\title{
Connections between Planck's and Gravitational Parameters
}

\author{
Branko M. Novakovic
}

\begin{abstract}
New Relativistic Alpha Field Theory (RAFT) extends GR to the extremally strong gravitational field, including Planck scale. In this theory, for spherically symmetric Planck mass, the minimal gravitational length (diameter) is equal to Planck length. Thus, minimal gravitational radius of Planck mass is equal to half of Planck length. The ratio of Planck mass and Planck length is constant, $M_{p} / L_{p}=c^{2} / G$. Generally, in gravitational field, the ratio of mass $M$ and minimal Gravitational length $L_{g}$ is also constant, $M / L_{g}=c^{2} / G$, and the same as the ratio of Planck mass and length. This fact is employed in this paper for derivation of the connections between Planck's and gravitational parameters. In that sense, the Gravitational length, time, energy and temperature are presented as the function of the Planck length, time, energy and temperature, respectively. This opens possibility for application of QFT to the gravitational field. The existence of a minimal radius in gravitational field means that no singularity in that field. Since the most minimal gravitational radius belongs to the minimal mass, the Planck length is not the shortest meaningful length in space-time.
\end{abstract}

Index Terms- Planck length, Gravitational length, Relativistic Alpha Field Theory (RAFT), extremely strong gravitational field.

\section{INTRODUCTION}

Following GRT [1-5], recently it has been developed a new Relativistic Alpha Field Theory (RAFT) [6-10]. This theory has a capability to extend application of GRT to the extremally strong gravitational field, including Planck's scale. In that sense, this theory offers the following predictions: a) there exists a minimal gravitational radius at $r_{\min }=G M / 2 c^{2}, \mathrm{~b}$ ) no singularity in a gravitational field, c) there exists a repulsive (positive) gravitational force in the region $G M / 2 c^{2}<r<G M / c^{2}, \mathrm{~d}$ ) at the radius $r=G M / c^{2}$ gravitational force is equal to zero, e) at radius $r>G M / c^{2}$ gravitational force is attractive (negative) as we know from experience, $\mathrm{f}$ ) the repulsive gravitational force could be a source of the so-called Dark Energy. Following the predictions of RAF theory, we can see that the region of the extremally strong gravitational field offers some non-expected answers in classical GR theory. Meanwhile, it is the consequence of the solution of the field equations by including gravitational energy-momentum tensor (EMT) on the right side of the field equations [11].

Following the existence of the minimal gravitational radius, in this paper we presented the related connections between Planck's and gravitational parameters. In that sense, we introduce some new notions in a gravitational field. At the first we introduce the Gravitational length as the function of

Branko Novakovic, FSB - University of Zagreb, Luciceva 5, P.O.B. 509, 10000 Zagreb, Croatia. the Planck length. It follows the Gravitational time as function of the Planck time, Gravitational energy as function of Planck energy and Gravitational temperature as function of Planck temperature. Further, this presentation also opens the possibility for application of QFT to the gravitational field. Classical approach to quantisation of gravitational field is presented in [12].

As we know, the Planck's length, $L_{p}$, [13-18] and Planck's mass, $M_{p}$, [19-21] are the base units in the system of natural units known as Planck's units that have been developed by physicist Max Planck. It is believed that the Planck length is the shortest meaningful length as the limiting distance below which the regular notions of space and length surcease to exist. There is currently no proven physical significance of the Planck's length. Meanwhile, it is theoretically considered to be the quantization of space in the quantum gravity theory [16]. In some forms of quantum gravity, the Planck's length is the length scale at which the structure of spacetime becomes dominated by quantum effects, and it is impossible to determine the difference between two locations less than one Planck' length apart. Any attempt to investigate the possible existence of smaller distances, by using higher energy collisions, would result in black hole production [15]. This is the consequence of the vacuum solution of the Einstein's field equations that predicts the singularities in the extremely strong field and the existence of the related black holes. Therefore, General Relativity Theory (GRT) [1-5] with vacuum solution of the Einstein's field equations cannot be applied to the extremely strong field at Planck's scale. But these problems can be satisfactory solved by application before mentioned RAF theory [6-10].

Following RAF theory, in the section II we introduce the Minimal Length Theorem (MLT). This theorem is valid for all spherically symmetric bodies (particles) in a gravitational field with mass $M$, including Planck mass $M_{p}$. Namely, the ratio of mass and its minimal length is equal to the constant $c^{2} / G$. Here $c$ is a speed of light in a vacuum and $G$ is gravitational constant. Thus, in this section we used the mentioned MLT in derivation of the Gravitational length as a function of Planck length.

In the section III we present the connection between Planck time $t_{p}$ and Gravitational time $t_{g}$. While Planck time is defined as time needed for the light to pass through one Planck length, the Gravitational time is the time needed for the light to pass through one Gravitational length. This connection gives the relation in which Gravitational time is a function of the Planck time.

In the section IV we derived connection between Planck energy $E_{p}$ end Gravitational energy $E_{g}$. As we know, Planck energy is in fact mass energy of the Planck mass $E_{p}=M_{p} c^{2}$. In that sense, Gravitational energy is equal to $E_{g}=M c^{2}$. Thus, this connection gives the relation in which Gravitational energy is a function of the Planck energy. 
The connection between Planck temperature $T_{p}$ and Gravitational temperature $T_{g}$ is presented in the section $\mathrm{V}$. While he Planck temperature is defined as the ratio of the Planck energy and Boltzmann constant $k$, the Gravitational temperature is defined as the ratio of the Gravitational energy and Boltzmann constant $k$. Thus, this connection gives the relation in which Gravitational temperature is a function of the Planck temperature.

Finally, in the section VI we presented the comparison of Planck and Gravitational parameters. In that sense, we put together the all of the connections between Planck's and Gravitational parameters. In all connected relations we have that Gravitational items (length, time, energy and temperature) are proportional to the related Planck's items (length, time, energy and temperature, respectively). Factor of proportionality is the ratio of gravitational mass and Planck mass $\left(\mathrm{M} / \mathrm{M}_{\mathrm{p}}\right)$. From the mentioned connections we can derive very important discoveries:

a) For all gravitational masses $M$ less than Planck mass $M_{p}$, the Gravitational items (length, time, energy and temperature) are less than the related Planck's items (length, time, energy and temperature, respectively).

b) For all gravitational masses $\mathbf{M}$ greater than Planck mass $\mathrm{M}_{\mathrm{p}}$, the Gravitational items (length, time, energy and temperature) are greater than the related Planck's items (length, time, energy and temperature, respectively).

This means that the Planck length is not the smallest length in a spacetime as the limiting distance below which any regular smaller distance in a gravitational field cannot exist. In fact, the smallest length in a spacetime belongs to the smallest gravitational mass. As an example, we calculated the Gravitational items (length, time, energy and temperature) for mass of proton. Since, the proton mass is less then Planck mass the all Gravitational items of proton are less than related Planck items. On the other hand, we also calculated the Gravitational items of the estimated Universe mass, as an example for the case where gravitational mass is much greater than Planck mass. This confirms the previous conclusion for the gravitational masses greater than Planck mass.

Further, the example with Universe mass maybe will result with a very important discovery: between two quantum states maybe there exist the transition process [11]. For the elementary particles this process is too small and can be neglected. But for the bigger masses the transition process maybe could not be allowed to be neglected. Thus, the calculation of the Gravitational time (in the first transition process) for the Universe mass showed very large amount of a time.

Furthermore, the existence of a non-singular rotating black hole, presented in [22], could also be one of the consequences of the existence of the minimal gravitational radius. In [23-31] different problems have been considered by applying Planck's parameters $\left(c, \hbar, G, L_{p}, M_{p}\right)$. The mentioned problems can also be solved by using maximal radial density in the gravitational field, $\rho_{\text {rmax }}=M / r_{\text {min }}=2 c^{2} / G$, that has been presented in [7]. The maximal radial density is the constant and the same for all gravitational mass.

\section{Connections Between Planck Length And Minimal LENGTH OF GRAVITATIONAL MASS}

As it is the well-known in physics, the Planck length, denoted by $L_{p}$, and Planck mass, denoted by $M_{p}$, are the units of length and mass, respectively, in the system of natural units known as Planck units [13,14,18,19,21]. To this system also belong Planck time $t_{p}$, Planck energy $E_{p}$ and Planck temperature $T_{p}$, derived as related function of Planck length and Planck mass.

In this section we derived Gravitational length $L_{g}$ as the function of the Planck length. In that sense we introduced a new Minimal Length Theorem (MLT).

Minimal Length Theorem (MLT). The all spherically symmetric bodies (particles) in a gravitational field with mass $M$, including Planck Mass $M_{p}$, should satisfies the following relation

$$
\begin{gathered}
\frac{M}{L_{\text {min }}}=\frac{M_{p}}{L_{p}}=\frac{c^{2}}{G}=\text { const. } \rightarrow L_{p}=2 r_{p}, L_{\text {min }}=2 r_{m}, \\
\rho_{\text {rmax }}=\frac{M_{p}}{r_{p}}=\frac{M}{r_{\text {min }}}=\frac{2 c^{2}}{G}=\text { const } .
\end{gathered}
$$

Here $L_{\min }$ and $r_{m}$ are minimal length and minimal radius of mass $M, L_{p}$ and $r_{p}$ are Planck length and Planck radius of Planck mass $M_{p}, c$ is a speed of light in vacuum and $G$ is gravitational constant. Here $L_{p}$ and $r_{p}$ are also minimal length and minimal radius of Planck mass. Further, $\rho_{r \text { max }}$ is the maximal radial density in a gravitational field that is constant and is valid for all masses in a space time. This is the prediction of RAF theory [6-8].

\section{Proof of the Minimal Length Theorem.}

Following recently developed Relativistic Alpha Field Theory (RAFT), the solution of the line element $d s$ for the gravitational spherically symmetric static field of mass $M$ gives the relation [6-8]

$$
\begin{aligned}
d s^{2}= & -\left(1-\frac{G M}{r c^{2}}\right)^{2} c^{2} d t^{2}+2 \sqrt{\frac{2 G M}{r c^{2}}-\left(\frac{G M}{r c^{2}}\right)^{2}} c d t d r \\
& +d r^{2}+r^{2} d \theta^{2}+r^{2} \sin ^{2} \theta d \phi^{2}
\end{aligned}
$$

Here $G$ is gravitational constant, $r$ is gravitational radius, $t$ is coordinate time, $c$ is speed of the light in vacuum, while $\theta$ and $\phi$ are related angles of spherically polar coordinates.

Remarks 1. The field parameters in the line element (2) satisfies the Einstein's field equations [1,2] with a cosmological constant $\Lambda=0$ and generate the related gravitational energy-momentum tensor $T_{\mu \eta}$ [11]. For that case, we do not need to add by hand the related energy-momentum tensor $T_{\mu \eta}$ on the right side of the Einstein's field equations. Thus, in the case of a strong static gravitational field, the quadratic term $\left(G M / r c^{2}\right)^{2}$ in (2) generates the related gravitational energy-momentum tensor $T_{\mu \eta}$. In the case of a relatively weak static gravitational field, like in our solar system, the quadratic term $\left(G M / r c^{2}\right)^{2}$ is too small and can be neglected. For that case, the field parameters (2) satisfy the Einstein's field equations in a vacuum $\left(T_{\mu \eta}=\right.$ 
$0, \Lambda=0$ ), and line element (2) is converted into the related vacuum solution

$$
\begin{aligned}
d s^{2}= & -\left(1-\frac{2 G M}{r c^{2}}\right) c^{2} d t^{2}+2 \sqrt{\frac{2 G M}{r c^{2}}} c d t d r \\
& +d r^{2}+r^{2} d \theta^{2}+r^{2} \sin ^{2} \theta d \phi^{2} .
\end{aligned}
$$

Following the solution of the line element (2) one can conclude that RAF theory extends GR to the extremely strong gravitational field including Planck's scale.

In order to find out the existence of the minimal radius in a gravitational field we can start with the line element (2). This line element can be rewritten into the following form

$$
\begin{aligned}
d s^{2}= & -\left(1-\frac{G M}{r c^{2}}\right)^{2} c^{2} d t^{2}+2 \sqrt{\frac{2 G M}{r c^{2}}\left(1-\frac{G M}{2 r c^{2}}\right)} c d t d r \\
& +d r^{2}+r^{2} d \theta^{2}+r^{2} \sin ^{2} \theta d \phi^{2}
\end{aligned}
$$

It is easy to prove that the line element (4) is regular if the following conditions are satisfied:

$$
\left(1-\frac{G M}{2 r c^{2}}\right) \geq 0 \rightarrow \frac{G M}{2 r c^{2}} \leq 1 \rightarrow \frac{G M}{2 c^{2}} \leq r<\infty .
$$

From the previous relation we can conclude that there exists the minimal gravitational radius $r_{m}$ which still preserves regularity of the line element (4)

$$
r_{m}=\frac{G M}{2 c^{2}} \text {. }
$$

For the radiuses less than minimal gravitational radius $r_{m}$ the line element (4) becomes imaginary item.

Now, applying of the Planck mass $M_{p}$ to the relation (6) we obtain minimal gravitational radius of the Planck mass

$$
r_{p m}=\frac{G M_{p}}{2 c^{2}} .
$$

As it is well-known, the Planck mass $M_{p}$ [19-21] and the Planck length $L_{p}$ [13-18] are defined from three fundamental physical constants: the speed of light in vacuum $c$, the reduced Planck constant $\hbar$ and the gravitational constant $G$ :

$$
M_{p}=\sqrt{\frac{\hbar c}{G}}, \quad L_{p}=\sqrt{\frac{\hbar G}{c^{3}}} .
$$

Assuming that the Planck mass $M_{p}$ has spherically symmetric form (i.e. $L_{p}$ is diameter of the Planck mass) and following the relations (7) and (8), we can calculate the following equality:

$$
\begin{aligned}
\frac{M_{p}}{2 r_{p m}} & =\frac{c^{2}}{G}, \quad \frac{M_{p}}{L_{p}}=\frac{\sqrt{\hbar c / G}}{\sqrt{\hbar G / c^{3}}}=\frac{c^{2}}{G}=\text { const. } \\
& \rightarrow 2 r_{p m}=L_{p} \rightarrow r_{p m}=\frac{L_{p}}{2} .
\end{aligned}
$$

Thus, the minimal gravitational radius of the Planck mass $M_{p}$ , denoted by $r_{p m}$, is equal to half of the Planck length $L_{p}$. This confirms that Planck length is minimal diameter of the Planck mass. It could be a physical meaning of the Planck length.

Now, assuming that mass $\mathrm{M}$ is spherically symmetric body, we can introduce Gravitational length $L_{g}$ as the minimal length in a gravitational field, generally:

$$
r_{m}=\frac{L_{g}}{2} \rightarrow L_{g}=2 r_{m}
$$

From the previous relations we can derive the following equations:

$$
\frac{L_{g}}{L_{p}}=\frac{M}{M_{p}} \rightarrow L_{g}=L_{p} \frac{M}{M_{p}}=\sqrt{\hbar G / c^{3}} \frac{M}{M_{p}} .
$$

The relations in (11) presented connection between Planck length $L_{p}$ end minimal gravitational length $L_{g}$. If the gravitational mass $M$ is equal to Planck's mass $M_{p}$, then Gravitational length $L_{g}$ is equal to the Planck length $L_{p}$. Now from (11) we can obtain the relation of the MLT (1):

$$
\left(\frac{L_{g}}{L_{p}}=\frac{M}{M_{p}}\right) \frac{M_{p}}{L_{g}} \rightarrow \frac{M_{p}}{L_{p}}=\frac{M}{L_{g}} .
$$

Thus, from the relations in (11a) we obtain relation (1) that proves the Minimal Length Theorem in (1).

Further, from the previous relations we can conclude that the minimal length in gravitational field is proportional to the mass. Thus, the most minimal lent in a gravitational field belongs to the minimal mass. Since the Planck mass is not the minimal mass in space-time, the Planck length is not the minimal length in the universe. This is very important discovery for the new investigations in physics. The relations in (11) can also be employed for quantization of a gravitational field by using the Planck length.

\section{CONNECTIONS BETWEEN PLANCK's TIME AND GRAVITATIONAL TIME}

As we know, Planck time is defined as the time needed for the light to pass through one Planck length

$$
t_{p}=\frac{L_{p}}{c}=\sqrt{\hbar G / c^{5}} .
$$

Following the relations (11) and (12), we can derive definition for the Gravitational time. Thus, the Gravitational time is the time needed for the light to pass through one Gravitational length

$$
t_{g}=\frac{L_{g}}{c}=\frac{L_{p}}{c} \frac{M}{M_{p}}=t_{p} \frac{M}{M_{p}}=\sqrt{\hbar G / c^{5}} \frac{M}{M_{p}} .
$$

The relations in (13) present connection between Planck time $t_{p}$ end the Gravitational time $t_{g}$. If the gravitational mass $M$ is equal to Planck mass $M_{p}$, then Gravitational time $t_{g}$ is equal to the Planck time $t_{p}$.

Further, from the relations in (13), we can conclude that the Gravitational time is proportional to the mass. Thus, the minimal time in a gravitational field belongs to the minimal mass. Since the Planck mass is not the minimal mass in space-time, the Planck time is not the minimal time in the universe. The relations in (13) can also be employed for quantization of a gravitational field by using the Planck time. 


\section{CONNECTIONS BETwEen Planck ENERGy AND GRAVITATIONAL ENERGY}

The Planck energy is defined as the mass energy of the Planck mass:

$$
\begin{gathered}
E_{p}=M_{p} c^{2}=\sqrt{\frac{\hbar c}{G}} c^{2}=\sqrt{\frac{\hbar c^{5}}{G}}, \\
E_{p}=\frac{\hbar}{t_{p}}=\hbar / \sqrt{\frac{\hbar G}{c^{5}}}=\sqrt{\frac{\hbar c^{5}}{G}}, \\
M_{p} c^{2}=\frac{\hbar}{t_{p}}, M_{p} t_{p}=\frac{\hbar}{c^{2}}, c=\sqrt{\frac{\hbar}{M_{p} t_{p}}} .
\end{gathered}
$$

Following the relations in (14), we can derive definition for the Gravitational energy. Thus, the Gravitational energy is defined as the mass energy of the Gravitational mass:

$$
\begin{gathered}
E_{p}=M_{p} c^{2}=\sqrt{\frac{\hbar c}{G}} c^{2}=\sqrt{\frac{\hbar c^{5}}{G}}, \quad c^{2}=\sqrt{\frac{\hbar c^{5}}{G}} \frac{1}{M_{p}}, \\
E_{g}=M c^{2}=\sqrt{\frac{\hbar c^{5}}{G}} \frac{M}{M_{p}}=E_{p} \frac{M}{M_{p}} .
\end{gathered}
$$

The relations in (15) presented connection between Planck energy $E_{p}$ end the Gravitational energy $E_{g}$. If the gravitational mass $M$ is equal to Planck mass $M_{p}$, then Gravitational energy $E_{g}$ is equal to the Planck energy $E_{p}$.

Further, from the relations in (15), we can conclude that the Gravitational energy is proportional to the mass. Thus, the minimal energy in a gravitational field belongs to the minimal mass. Since the Planck mass is not the minimal mass in space-time, the Planck energy is not the minimal energy in the universe. The relations in (15) can also be employed for quantization of a gravitational field by using the Planck energy.

\section{CONNECTIONS BETWEEN PLANCK TEMPERATURE AND GRAVITATIONAL TEMPERATURE}

The Planck temperature is defined as the ratio of the Planck energy and Boltzmann constant $k$

$$
T_{p}=\frac{E_{p}}{k}=\frac{M_{p} c^{2}}{k}=\sqrt{\frac{\hbar c^{5}}{G k^{2}}} .
$$

Following the relation (16), we can derive definition for the Gravitational temperature. Thus, the Gravitational temperature is the ratio of the Gravitational energy and Boltzmann constant $k$

$$
T_{g}=\frac{E_{g}}{k}=\frac{M c^{2}}{k}=\sqrt{\frac{\hbar c^{5}}{G k}} \frac{M}{M_{p}}=T_{p} \frac{M}{M_{p}} .
$$

The relations in (17) presented connection between Planck temperature $T_{p}$ end the Gravitational temperature $T_{g}$. If the gravitational mass $M$ is equal to Planck mass $M_{p}$, then Gravitational temperature $T_{g}$ is equal to the Planck temperature $T_{p}$.
Further, from the relations in (17), we can conclude that the Gravitational temperature is proportional to the mass. Thus, the minimal temperature in a gravitational field belongs to the minimal mass. Since the Planck mass is not the minimal mass in space-time, the Planck temperature is not the minimal temperature in the space-time. The relations in (17) can also be employed for quantization of a gravitational field by using the Planck temperature.

\section{COMPARISON OF PLANCK'S AND GRAVITATIONAL PARAMETERS}

Following the relations $(11,13,15,17)$ we can put together the all of the connections between Planck's and Gravitational parameters. At the first, we have the connections between Planck length $L_{p}$ and Planck time $t_{p}$ with Gravitational length $L_{g}$ and Gravitational time $t_{g}$ :

$$
\begin{aligned}
& L_{g}=L_{p} \frac{M}{M_{p}}=\sqrt{\hbar G / c^{3}} \frac{M}{M_{p}}, \\
& t_{g}=t_{p} \frac{M}{M_{p}}=\sqrt{\hbar G / c^{5}} \frac{M}{M_{p}} .
\end{aligned}
$$

It follows the connections between Planck energy $E_{p}$ and Planck temperature $T_{p}$ with Gravitational energy $E_{g}$ and Gravitational temperature $T_{g}$ :

$$
\begin{gathered}
E_{g}=E_{p} \frac{M}{M_{p}}=\sqrt{\frac{\hbar c^{5}}{G}} \frac{M}{M_{p}}, \\
T_{g}=T_{p} \frac{M}{M_{p}}=\sqrt{\frac{\hbar c^{5}}{G k}} \frac{M}{M_{p}} .
\end{gathered}
$$

From the relations (18) and (19) we can conclude the following: The all gravitational parameters can be calculated by multiplying the related Planck's parameters with the ratio of gravitational mass and Planck mass $\left(M / M_{p}\right)$.

Now we can calculate the applications of previous results to the particle proton. The amounts of the Planck's parameters and mass of the proton $M_{p r}$ [14,19] are given with the relations:

$$
\begin{gathered}
M_{p}=2.176435 \cdot 10^{-8} \mathrm{~kg}, L_{p}=1.616255 \cdot 10^{-35} \mathrm{~m}, \\
t_{p}=5.391245 \cdot 10^{-44} \mathrm{~s}, E_{p}=1,956 \cdot 10^{9} \mathrm{~J}, \\
T_{p}=1.416808 \cdot 10^{32} \mathrm{~K}, M_{p r}=1.672622 \cdot 10^{-27} \mathrm{~kg} .
\end{gathered}
$$

In order to simplify the calculations, we used maximal six decimals in (20). Applying (20) to the relations (18) and (19) we obtain the following result for the proton:

$$
\begin{aligned}
& L_{g p r}=1.242116 \cdot 10^{-54} \mathrm{~m}, t_{g p r}=4.143253 \cdot 10^{-63} \mathrm{~s}, \\
& E_{g p r}=1.503215 \cdot 10^{-10} \mathrm{~J}, T_{g p r}=1.088838 \cdot 10^{13} \mathrm{~K} .
\end{aligned}
$$

Compare the gravitational parameters of the proton in (21) with the related Planck's parameters in (20) we can obtain very important conclusions. At the first, Gravitational length of the proton is less than Planck length. This confirms that Planck length is not the meaningful minimal length in space-time, because the minimal length belongs to the minimal mass. Further, the Gravitational time of the proton is less than Planck time. This is the consequence of the fact that 
the Gravitational length of the proton is less than Planck length.

Generally, following the relations in (20) and (21), we can conclude that the all Gravitational parameters for the masses less than Planck mass are smaller than the related Planck's parameters. On the contrary, the all Gravitational parameters for the masses greater than Planck mass are larger than the related Planck's parameters.

Further, we calculated the applications of previous results to the Universe. The estimated total mass $M_{u}$ and radius $r_{u}$ of the visible Universe is given here [19]:

$$
M_{u}=1.5 \cdot 10^{53} \mathrm{~kg}, \quad r_{u}=4.4 \cdot 10^{26} \mathrm{~m} .
$$

Applying Planck's parameters from (20) and Universe parameters from (22) to the relations (18) and (19) we obtain the following results for the Universe:

$$
\begin{gathered}
L_{g u}=1.113923 \cdot 10^{26} \mathrm{~m}, t_{g u}=11.782236 \cdot 10^{9} \text { years }, \\
E_{g u}=13.480752 \cdot 10^{69} \mathrm{~J}, T_{g u}=9.764641 \cdot 10^{92} \mathrm{~K}, \\
r_{g u}=L_{g u} / 2=5.569615 \cdot 10^{25} \mathrm{~m} .
\end{gathered}
$$

In order to compare present radius of the Universe (22) with its minimal radius (23) we have to consider the radial velocity and radial acceleration of the universe expansion [9]:

$$
\begin{gathered}
\dot{r}= \pm\left[\frac{2 G M}{r}\left(1-\frac{G M}{2 r c^{2}}\right)+\left(\kappa^{2}-1\right) c^{2}\right]^{1 / 2}, \\
\ddot{r}=-\frac{G M}{r^{2}}\left(1-\frac{G M}{r c^{2}}\right) .
\end{gathered}
$$

Here $\kappa$ is the energy conservation constant. At the moment we do not know exactly amount of this constant. But we know that this constant is close to unity bat little bit less than unity. In this presentation we will assume that $\kappa=1$. In that case the first relation in (24) is simplified:

$$
\begin{gathered}
\kappa=1 \rightarrow \dot{r}= \pm\left[\frac{2 G M}{r}\left(1-\frac{G M}{2 r c^{2}}\right)\right]^{1 / 2}, \\
\ddot{r}=-\frac{G M}{r^{2}}\left(1-\frac{G M}{r c^{2}}\right) .
\end{gathered}
$$

At the minimal gravitational radius, the Universe velocity and acceleration have the following amounts:

$$
r=\frac{G M}{2 c^{2}} \rightarrow \dot{r}=0, \quad \ddot{r}=\frac{4 c^{2}}{G M}=\ddot{r}_{\text {max }} .
$$

Thus, at the minimal gravitational radius radial velocity is equal to zero, and radial acceleration is positive (repulsive). This is the necessary condition for starting with accelerated expansion.

At the radius $r_{c}=G M / c^{2}$, the Universe velocity and acceleration have the amounts:

$$
r=\frac{G M}{c^{2}} \rightarrow \dot{r}_{c}=c=\dot{r}_{c_{\max }}, \quad \ddot{r}_{c}=0 .
$$

Thus, at the gravitational radius $r_{c}$, radial velocity is maximal, and radial acceleration is equal to zero. For radius greater than $r_{c}$, the radial velocity is less than $c$ and the radial acceleration becomes negative (attractive). This is the necessary condition for starting with further expansion, but now with deceleration.

From observation of the visible Universe, we know that present expansion velocity is less then $c$ and the Universe expansion is with the related acceleration. This means that present radius of the Universe $r_{p}$ should be between minimal radius $r_{\min }$ and radius $r_{c}$ :

$$
\begin{gathered}
r_{\text {min }}<r_{p}<r_{c}=2 r_{\text {min }}=L_{g u} \rightarrow \\
5.569615 \cdot 10^{25} m<r_{p}<1.113923 \cdot 10^{26} \mathrm{~m} .
\end{gathered}
$$

Since the observed expansion velocity is close to $c$, we can conclude that present Universe radius should be close to $L_{g u}$. Thus, if the observed Universe mass is correct, then the calculated present universe radius (28) is about four times less than observed radius (22).

In the opposite case, if the observed Universe radius is correct, then the present universe mass can be calculated as follow:

$$
\begin{gathered}
\frac{M}{L_{\text {min }}}=\frac{c^{2}}{G} \rightarrow M=L_{\text {min }} \frac{c^{2}}{G}, \\
L_{\text {min }}=2 r_{u}=8.8 \cdot 10^{26} \mathrm{~m}, M_{c a l}=11.85 \cdot 10^{53} \mathrm{~kg}, \\
M_{c a l} / M_{o b s}=7.9 \approx 8 .
\end{gathered}
$$

Thus, the calculated Universe mass is about eight times greater than observed Universe mass.

\section{CONCLUSION}

Following Relativistic Alpha Field Theory (RAFT), that extends GR to the extremally strong gravitational field, we elaborated the possibility of the connections between Planck's and gravitational parameters. In that sense, the Gravitational length, time, energy and temperature are presented as the function of the Planck's length, time, energy and temperature, respectively. This also opens possibility for application of QFT to the gravitational field. The previous conclusion is the consequence of the existence of a minimal radius in gravitational field predicted by RAF theory. This also means that no singularity in a gravitational field. It is because at the minimal gravitational radius maximal radial density is equal to $M / r=c^{2} / 2 G$, the gravitation acceleration is positive, gravitational velocity is equal to zero and a gravitational force becomes repulsive. Thus, at the minimal gravitational radius we have the satisfactory conditions for starting with repulsive expansion.

\section{REFERENCES}

[1] Einstein, A., Ann. Phys. 49, 769-822 (1916).

[2] Einstein, A.: The Meaning of Relativity. Princeton Univ. Press, Princeton (1955)

[3] Sean.C.: Spacetime and geometry: An introduction to general relativity. Amazon,com Books.htm, Hardcover (2003).

[4] Weinberg, S.: Gravitational and cosmology: Principles and application of the general relativity. Gebundene ausgabe,

[5] Hawking, S. W., Ellis, G. F. R.: The Large Scale Structure of Space-Time. Univ. Press, Cambridge (1973). 
[6] Novakovic, B. M.: Relativistic alpha field theory - Part I: Determination of Field Parameters. International Journal of New Technology and Research (IJNTR) ISSN:2454-4116, 1, 5 (2015) 23-30. https://doi.org/10.31871/IJNTR.1.5.15

[7] Novakovic, B. M.: Relativistic alpha field theory-Part II: Does a Gravitational Field Could be Without Singularity? International Journal of New Technology and Research (IJNTR) ISSN:2454-4116, 1, 5 (2015) 31-38 https://doi.org/10.31871/IJNTR.1.5.16

[8] Novakovic, B. M.: Relativistic alpha field theory-Part III: Does Gravitational Force Becomes Positive if $\left(\mathrm{GM} / \mathrm{rc}^{2}\right)>1$ ? International Journal of New Technology and Research (IJNTR) ISSN:2454-4116, 1, 5 (2015) 39-47. https://doi.org/10.31871/IJNTR.1.5.17

[9] Novakovic, B. M. Is positive Gravitational Force Source of Dark Energy?, International Journal of New Technology and Research (IJNTR) ISSN:2454-4116, 1, 7 (2015) 6-13. https://doi.org/10.31871/IJNTR.1.7.5

[10] Novakovic, B. M.: Generalized Relativistic Hamiltonian in an Alpha Field. AIP Conference Proceedings 1303, 141 (2010). https://doi.org/10.1063/1.3527149

[11] Novakovic, B. M.: Physical Properties of Energy-Momentum Tensor of Gravitational Field in RAF Theory. International Journal of New Technology and Research (IJNTR) ISSN:2454-4116, 4, 11 (2018) 19-25.

https://doi.org/10.31871/IJNTR.4.11.10

[12] Novakovic, B. M.: Quantum Gravity in Relativistic Alpha Field Theory (QG in RAFT). International Journal of New Technology and Research (IJNTR) ISSN:2454-4116, 4, 6 (2018) 26-33. https://doi.org/10.31871/IJNTR.4.6.4

[13] Baez, J.: The Planck length. (1999). (http://math.ucr.edu/home/baez/planck/node2.html

[14] CODATA: Constants, Planck length. US National Institute of Standard and Technology, June (2015). (http://physics.nist.gov/cgi-bin/cuu/Value?plkl)

[15] Carr, B. J., Giddings, S. B.: Quantum Black Holes, Scientific American 292, 48-55 (2005).

[16] Garay, L. J.: Quantum gravity and minimum length, International Journal of Modern Physics, A 10, 145 (1995). doi: $10.1142 / \mathrm{S} 0217751 \mathrm{X} 95000085$

[17] Burges, C., Quevedo, F.: The Grate Cosmic Roller-Coaster Ride Scientific American (print). Scientific American, Inc. 55 (2007).

[18] Bowley, R., Eaves, L.: Planck Length. (2010). (http://www.sixtysymbols.com/videos/plancklength.htm)

[19] CODATA Value: Planck mass. US National Institute of Standard and Technology, June (2015). (http://physics.nist.gov/cgi-bin/cuu/Value?plkm)

[20] Bergmann, P. G.: The riddle of gravitation. Courier Corporation (1992).

(https://books.google.com/books?id=WYxkrwMidp0C\&pg=PR10)

[21] Sivaram, C.: What is Special About the Planck Mass? arXiv:0707.0058v1 (2007).

[22] Perkins, D. H.: Introduction to High Energy Physics. Cambridge Univ. Press, Cambridge (2000).

[23] Sherman, D., et al. Nat. Phys. 11, 1-5 (2015).

[24] Meckel, M., et al. Nat. Phys. 10, 594 - 600 (2014)

[25] Torres, R. Gen Relativ Gravit (2017) 49: 74 https://doi.org/10.1007/s10714-017-2236-5

[26] Garecki, J. Gen Relativ Gravit (1993) 25: 257. https://doi.org/10.1007/BF00756260

[27] Manfredi, G. Gen Relativ Gravit (2015) 47:1. https://doi.org/10.1007/s10714-014-1846-4

[28] Schiffer, M. Gen Relativ Gravit (1992) 24: 705. https://doi.org/10.1007/BF00760077

[29] Arbab, A.I. General Relativity and Gravitation (2004) 36: 2465. https://doi.org/10.1023/B:GERG.0000046833.08113.6b

[30] De Lorenzo, T., Pacilio, C., Rovelli, C. et al. Gen Relativ Gravit (2015) 47: 41.https://doi.org/10.1007/s10714-015-1882-8

[31] Li, H.L., Chen, SR. Gen Relativ Gravit (2017) 49: 128. https://doi.org/10.1007/s10714-017-2296-6

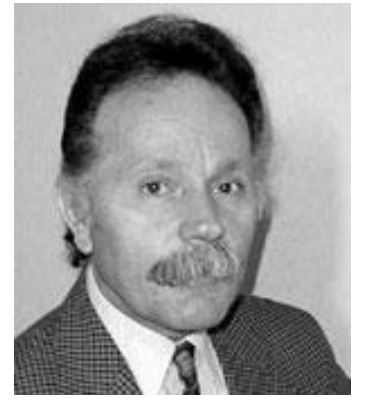

Branko Novakovic is a Professor emeritus at FSB - University of Zagreb, Croatia. Prof. Novakovic received his $\mathrm{PhD}$ from the University of Zagreb in 1978. His research of interest includes physics, control systems, robotics, neural networks, and fuzzy control. He is author of three books; Relativistic Alpha Field Theory (RAFT, e-book, 2016), Control Methods in Robotics, Flexible Manufacturing Systems and Processes (1990), Control Systems (1985) and the first co-author of a book Artificial Neural Networks (1998). He has published over 235 research papers in his research of interest. 